Copyright Agency Limited (CAL) licensed copy. Further copying and Communication prohibited except on payment of fee per Copy or Commuication And otherwise in accordance with the licence from CAL to ACER. For more Information contact CAL on (02) 9394-7600 or info@copyright contau

David Shum, Rochelle Matjac and Heather Ward

School of Psychology, Griffith University

\title{
Early developmental trends on time- and event-based prospective memory tasks
}

\begin{abstract}
This study assessed childhood prospective memory, the memory for future intentions such as remembering to hand in homework by: (a) comparing 47 children in three age groups; (b) using both timem (i.e., CyberCruiser) *, and event-based (i.e., a card sorting task) prospective memory tasks; and (c) examining relationships between prospective and retrospective memory, and prospective memory and two tests of executive function (Stroop Colour-Word Interference Test [Stroop] and Tower of London [TOL]. Results indicated improvements with age, albeit not identical patterns of improvement, on both prospective- and retrospective-memory tasks and the TOL. Furthermore, the TOL was significantly correlated with both measures of prospective memory. The different patterns of improvement with age on the prospective memory tasks suggest that it is not the type of task per se that matters so much as the complexity of the task. It is recommended that aspects of task demand be investigated further.
\end{abstract}

* The authors wish to thank Kimberley Kerns for providing a copy of CyberCruiser, the task she designed.

Keywords: Prospective memory, Retrospective memory, Children, Executive functions.

Prospective memory involves the integration of processes and skills required to fulfil a preplanned future action (Ellis \& Kvavilashvili, 2000) such as remembering to attend an appointment tomorrow. Event-based tasks require an individual to perform an action when an external event or stimulus appears. This external event acts as a cue for memory intentions (Einstein \& McDaniel, 1990; Ellis, 1996; Kerns, 2000). For example, someone walking into a room prompts you to remember to pass on a message to that person. Time-based tasks require the individual to perform some action at a specific time. This form of memory is self-initiated because no external cues or stimuli are present to prompt remembering (Einstein \& McDaniel; Ellis; Kerns). Time-based tasks are often considered of a higher cognitive order than eventmbased tasks, and therefore more difficult because in addition to remembering the task, and remembering to act on the intention at the right time, individuals must also monitor the passing of time (Kerns). 
Despite the importance of prospective memory in childhood (e.g., remembering to take excursion money to school, and pass on school messages to parents), not many studies have addressed this issue in children. According to Kerns (2000), the studies that do exist have had measurement problems. For example, assessment has relied on very small numbers of responses, or on children's ability to read and understand a clock. Furthermore, Kvavilashvili, Messer, and Ebdon (2001) expressed concern that too many different variables have been used over a wide range of tasks. Therefore, Kerns and Kvavilashvili and associates, independently attempted to study childhood prospective memory more systematically than had been done previously.

Kerns's (2000) aims were to assess a wider age range than previously, assess time-based prospective memory without the need to read a clock, use multiple trials, and compare prospective memory performance with executivefunctioning to try to identify cognitive processes involved in prospective memory. Kvavilashvili and colleagues's (2001) aims were to manipulate the difficulty of an ongoing task in an event-based paradigm, and to compare the developmental trajectory of prospective memory with that of retrospective memory (i.e., memory for past facts and events).

Kerns (2000) developed a computer task called CyberCruiser. The task was for 6- to 12 year-olds to manoeuvre a vehicle through traffic and to accumulate as many points as possible. Points were gained for driving speed and precision. The prospective memory task was to refuel the vehicle when fuel levels dropped. If the car ran out of fuel, all points were lost. The dependent measures were the number of fuel run outs, and the number of fuel checks. Results showed that older children ran out of fuel less than younger children. However, younger children checked the fuel gauge as often as older children, suggesting that the younger children were less able to use the information gained from their monitoring as strategically as the older ones. In addition, Kerns compared performance on the time-based prospective tasks with performance on executive function tasks, Go-No-Go, Stroop, Delayed Alternation-Nonalternation and the Self-Ordered Pointing Task (SOPT). Results showed that, even after controlling for age, there were significant correlations between the number of fuel run outs, and components of the Delayed Alternation task, the SOPT and the Stroop.

Kvavilashvili et al.'s task (2001) featured playing cards with pictures of familiar objects. Children aged 4- to 7 years were asked to name the items pictured on 80 cards that were divided into 4 packs of 20 . Inserted in each pack was a picture of an animal, a cow, dog, pig, and horse respectively. These were the prospective memory targets. The children were told to place the animal cards in a box when they appeared. Half the children's animal cards were inserted in the middle of the packs, which required the naming task to be interrupted. The other half's were placed at the bottom of the packs, which did not require the naming task to be interrupted. The children were also asked to recall the pictures they had named in the last pack (retrospective memory task). Results showed that those in the interrupt condition remembered to place the animal cards in the box less than those in the non interrupt condition. 
However, although 7 year-olds performed better than 4- and 5myear-olds, the effects of age explained only 7 to $10 \%$ of the variability in prospective memory performance. Further, the Age $x$ Interruption interaction was not significant. By contrast, on the recall test, 4- and 5-year-olds remembered signficantly fewer items than the 7-year-olds.

Kerns (2000) demonstrated a clear developmental trajectory in time-based prospective memory, showed that monitoring was more strategic in older than younger children, and that overlaps exist between time-based prospective memory and executive functioning. Kvavilashvili and associates (2001) showed that on event-based tasks, 4-year-olds could remember almost as well as 7-yearolds, but that all children found it more difficult when they had to interrupt another task, suggesting that the more cognitive resources have to be shared between two mental tasks, the less likely children are to remember a prospective task. Finally, Kvavilashvili et al. showed that retrospective memory performance improved steadily with maturity from 4- to 7-yearsm-of-age, and because of its different trajectory, by implication, is a different construct from prospective memory.

The current study aimed to bring together the best features of Kerns's (2000) and Kvavilashvili and colleagues' (2001) studies by incorporating both Kerns's time- and components of Kvavilashvili and colleagues' event-based paradigms to see if the two tasks produced different age effects. Furthermore, Kerns's wide age range, 6 to 12 years, was employed, but the sample was divided into three subgroups to isolate where age differences lay because cognitive development is a rapid process in chilchood (Anderson, Northam, Hendy, \& Wrennell, 2001). This study also aimed to test the relationship between the time-based task and two measures of executive function (i.e., Stroop and TOL), one of which (viz., the Stroop), was used by Kerns, and to assess retrospective memory as done by Kvavilashvili et al.

Given the complexity of time-based tasks and that age-related improvements have been shown in previous studies of time-based tasks in children (e.g., Ceci \& Bronfenbrenner, 1985; Kerns, 2000), this research hypothesised that on the CyberCruiser, children aged 6 to 8 years would run out of fuel more often than children aged 9 to 10 years or 11 to 12 years, and that the 9 - to 10 -year-olds would run out more often than 11- to 12-year-olds. However, given the lack of age effects found in children's event-based tasks (Kvavilashvili et al., 2001), it was hypothesised that on the adaptation of the card-naming task, children in the 6-8 group would hide fewer target cards than children in the 9-10 and 11-12 groups, but that children in the 9-10 group and 11-12 group would not differ significantly because the processes involved in the event-based task should be wellestablished by middle childhood.

Evidence supports a relationship between prospective memory and executive functions (Maujean, Shum, \& McQueen, 2003; Kerns, 2000). Moreover, executive function performance also appears to parallel the developmental trajectory of prospective memory (Kerns). Therefore, it was hypothesised that children in the 6-8 group would be poorer on the Stroop (focused attention) and 
TOL (planning) than children in the 9-10 and 11-12 groups, and that children in the 9-10 group would be poorer than those in the 11-12 group. It was also hypothesised that there would be a significant positive relationship between Stroop and TOL scores and the time-based prospective memory scores, because all are relatively resource demanding. By contrast, it was hypothesised that the relationship between Stroop and TOL scores and event-based prospective memory scores would be weaker, because the latter is less resource demanding, And finally, it was hypothesied that the Stroop and TOL scores would not correlate significantly with those of the recall task.

\section{Method}

\section{Participants}

Participants were 47 students from a primary school in the south-eastern region of Queensland. All children were reported by their parents to be free of psychological, neurological, and communication problems. Estimates of the children's IQs were obtained using two subtests: Vocabulary and Martrix Reasoning, of the Wecshler Abbreviated Scale of Intelligence (WASI, The Psychological Corporation, 1999). Table 1 shows the children's mean ages, estimated IQs and standard deviations by group and gender.

\section{Materials and tasks}

Time-based prospective memory task

The CyberCruiser (Kerns, 2000) software was loaded onto a notebook computer and the children interacted with the program via a Blackhawk Mark 2 joystick. In the ongoing task, participants "drove" a car down a busy road towards oncoming traffic, accumulating as many points as possible. Points increased as driving speed increased, and decreased every time there was a collision with another vehicle. To keep the children motivated, the names of the three highest scorers and their points were displayed on the screen. The participants' own cumulative scores were also clearly visible.

Table I. Mean Ages, Estimated IQs, (and standard deviations) as a function of Group and Gender

\begin{tabular}{|lcccccc}
\hline & \multicolumn{2}{c}{ Group } & \multicolumn{2}{c}{ Group } & \multicolumn{2}{c}{ Group } \\
\hline & \multicolumn{2}{c}{$6-8$ Years } & \multicolumn{2}{c}{ 9-10 Years } & \multicolumn{2}{c}{$11-12$ Years } \\
\hline & Females & Males & Females & Males & Females & Males \\
\hline Mean age (yrs) & 6.86 & 7.38 & 9.6 & 9.83 & 11.56 & 11.86 \\
SD & $(0.38)$ & $(0.74)$ & $(0.52)$ & $(0.75)$ & $(0.53)$ & $(0.38)$ \\
\hline Mean est. IQ & 93.93 & 101.25 & 98.81 & & & \\
SD & $(6.09)$ & $(12.95)$ & $(10.22)$ & & & \\
\hline
\end{tabular}


The prospective task was to refuel the car. The importance of checking the fuel was stressed and the children were warned that if they ran out, all points would be lost and they would have to restart the game. The fuel gauge appeared on the bottom of the screen, but only when the player pressed a button on the joystick. The gauge only remained in view for 3 seconds so not to cue memory. Refuelling involved pressing the same button on the joystick, but only when the needle on the gauge was in the red zone. An alarm sounded if the car ran out of fuel and a message appeared on the screen stating: "You ran out of fuel, start again". The car was then refuelled, all points lost and the game restarted.

The game ran for 5 minutes. If the car was not refuelled within a 1-minute interval of play, a fuel run out occurred. Two dependent measures were obtained, the number of fuel run outs, and the number of fuel gauge checks made in 15 second intervals.

After completing the game, to ensure prospective memory failures were not due to retrospective memory difficulties, the children were asked to recall the task instructions.

\section{Event-based prospective memory task}

This task, adapted from Kvavilashvili et al. (2001), utilised coloured crayons, blank sheets of paper, a toy bear, and 80 laminated, white cardboard cards $(12.5 \mathrm{~cm} \times 9 \mathrm{~cm})$. Each of the cards showed a colour picture of an animal, which were selected from Microsoft Master Clips Browser. The animal pictures comprised four animal categories: sea creatures, farm and domestic animals, exotic animals, and birds. The cards were randomly divided into four packs, each consisting of 20 cards and containing equal numbers from each category. The four animal cards used by Kvavilashvili et al. were again used as prospective-memory targets (i.e., a horse, cow, dog, and pig). One of the target cards was placed into each pack, always in the tenth position.

The children met the toy bear called "Jack", who had recently lost his glasses, and consequently could not see very well. The children were told that Jack owned the cards, but, because he got them just before losing his glasses, he did not know what the pictures were. The children were instructed to tell Jack what was on the cards.

The children were required, one pack at a time, to turn over each card, and to say the name of the animal that appeared. The prospective task was introduced when the experimenter noted that Jack was very scared of dogs, cows, horses, and pigs. So, if the children saw a picture of one of these animals, they were not to say its name, but, instead, place it in a box on a table behind them. The box was placed out of view so it did not act as an additional cue.

A filled delay followed the instructions. This ensured there was a reasonable interval between encoding and retrieving the prospective-memory intention (Ellis \& Kvavilashvili, 2000). The children drew a tree for Jack before the first pack of cards was sorted, then a house, car, and themselves before sorting the second, third and fourth packs of cards respectively. The dependent measure was the score out of four, for the number of cards hidden correctly. 
Post-Experimental Interview

In accordance with Kvavilashvili and colleagues (2001), to ensure that prospective-memory performance was not affected by retrospective memory failure, the children were asked to recall the instructions using three different prompts. The first prompt was, "Other than drawing pictures and naming the cards, was there anything else you were asked to do?" A second prompt was given if participants were unable to answer correctly: "Were you asked to do anything when you saw certain cards?". The third prompt was given if participants were still unable to answer: "What were you asked to do when you saw a picture of a dog, pig, horse, or cow?"

Retrospective memory task

After the last pack was sorted, children were asked to recall as many of the 20 animals as they could from the last pack. The dependent measure was the score out of 20 .

\section{Executive function tasks}

Stroop (Stroop, 1935). A standardized version of the Stroop as described by Golden, Espe-Pfeifer, and Wachsler-Felder (2000) was used as a measure of focused attention. The children read the names of colours ( $W$-block), named the colour of $X X X X_{s}$ printed in varying colours (C-block), and named the colour of the ink in which incongruent colour names were printed ( $\mathrm{CW}$-block) in 45 -s periods. After raw scores were age adjusted, interference scores were calculated by subtracting the number of items completed on the CW block from the number of items predicted to be completed using the formula: $(\mathrm{C} \times W) /(\mathrm{C}+\mathrm{W})$ (Golden, et al.). Interference scores were used as the dependent measure.

The Stroop has been found to: (a) have good test-retest reliability (.75 to .90, Uttl \& Graf, 1997); (b) to be moderately related to Perceptual Organisation ( $r=$ .37); and (c) to load on the same factor as serial subtraction tasks, and to reflect the ability to sustain mental processes (Shum, McFarland, \& Bain, 1990). The Stroop is also sensitive to brain injury (Hanes, Andrewes, Smith, \& Pantelis, 1996), and to age effects in 12- to 83-year-olds (Uttl \& Graf).

TOL. The TOL is well known as a measure of planning ability (Lezak, 1982; Shallice, 1982). This study used a version of the TOL developed by Tunstall (1999) who increased the number of disks from three to four to overcome a ceiling effect commonly reported in the original version (Levin, et al., 1997). Participants were required to rearrange the four different coloured disks on three poles of various heights to match a specific arrangement, and to do so in a specified number of moves. There were 10 problems, progressing from easy to more difficult. The children were allowed three attempts per problem. Three points were awarded for solving a problem on the first attempt, two on the second, and one on the third. Scores were summed across the 10 problems. The lowest possible score was 0 , and the highest was 30 . The total scores represented the dependent measure.

The 4-disk version of the TOL has been found to have adequate test-retest reliability (.60), to load on the same factor as the Porteus Mazes (Tunstall, 1999), 
and to be sensitive in detecting planning impairments in children with TBI (Shum, et al., 2000).

\section{Procedure}

Children were tested individually in a quiet room at their school. Tasks were administered in the following order: Vocabulary subtest of the WASI, CyberCruiser, card sorting, Stroop, Matrix Reasoning subtest of the WASI, and TOL.

\section{Results}

One-way ANOVAS were used to examine the impact of age on the dependent variables. All planned comparisons used one tailed $t$ tests calculated with MS error from the omnibus $F$ tests. To determine if there were gender differences, a series of $2 \times 3$ between-subjects ANOVAs was conducted. The independent variables were Group and Gender. There were several significant main effects for Group, with $F$ values reported separately below for each dependent measure. However, there were no main effects for Gender and no Group x Gender interactions. Because of the lack of gender effects, the scores for males and females were combined in all subsequent analyses.

Time- based task (CyberCruiser)

Table 2 shows the mean number of fuel run outs across the three age groups. A vistal inspection of the means shows that the 6-8 group ran out of fuel more than either the $9-10$ or $11-12$ groups, but that the $9-10$ and $11-12$ groups had similar run outs. A one-way ANOVA showed that overall group differences were significant, $F(2,44)=4.77, p<.05$. Results of planned comparisons revealed that 6- to 8-year-olds ran out of fuel significantly more than 9- to 10 year-olds, $t(44)=1.78, p<.05$, and 11 to 12 -year-olds, $t(44)=3.10, p<.01$. However, there was no difference in fuel run outs between the 9-10 and 11-12 groups, $t(44)=1.32, p>.05$.

Table 2 shows that the 6-8 group checked the fuel gauge slightly less than the 9-10 and 11-12 groups, but that there was little difference between the 9-10 and 11-12 groups. Results of a one-way ANOVA showed in fact that there were no significant group differences in checking behaviour, $F(2,44)=0.91, p>05$, and planned comparisons confirmed this. All children were able to remember the task instructions well, indicating that prospective forgetting was not due to retrospective forgetting.

\section{Event-based task (ard Sorting)}

Table 2 shows group comparisons on the mean number of cards hidden. A visual inspection of the means shows there were incremental improvements in remembering from 6- to 8-years, through 9- to 10-years, up to 11- to 12-years. A one-way ANOVA showed that overall these age differences were significant, $F(2$, $44)=8.97, \mathrm{p}<.01$. Planned comparisons indicated that the $6-8$ group did indeed remember to hide the cards significantly less than the 9 - 10 group, $t(44)=2.34, p<$ 
.05, and the 11-12 group, $t(44)=4.27, p<.001$. Furthermore, the $9 m 10$ group remembered significantly less than the $11-12$ group, $t(44)=1.93, p<.05$.

\begin{tabular}{|c|c|c|c|c|c|c|}
\hline \multirow[b]{3}{*}{ Measure } & \multicolumn{2}{|c|}{ Age Group } & \multicolumn{2}{|c|}{ Age Group } & \multicolumn{2}{|c|}{ Age Group } \\
\hline & \multicolumn{2}{|c|}{$6-8$} & \multicolumn{2}{|c|}{$9-10$} & \multicolumn{2}{|c|}{$11-12$} \\
\hline & M & SD & M & SD & $M$ & $S D$ \\
\hline \multicolumn{7}{|l|}{ PM* Tasks } \\
\hline Total fuel run outs & 3.40 & 1.30 & 2.63 & 1.09 & 2.06 & 1.24 \\
\hline $\begin{array}{l}\text { Number of fuel } \\
\text { checks }\end{array}$ & 15.07 & 9.15 & 19.13 & 9.34 & 17.62 & 6.60 \\
\hline $\begin{array}{l}\text { Number of cards } \\
\text { correctly hidden }\end{array}$ & 2.13 & 0.99 & 2.88 & 0.89 & 3.50 & 0.82 \\
\hline \multicolumn{7}{|l|}{ RM§ Tasks } \\
\hline Total cards recalled & 4.67 & 1.45 & 6.87 & 1.50 & 6.69 & 2.33 \\
\hline \multicolumn{7}{|l|}{ Executive Tasks } \\
\hline \multicolumn{7}{|l|}{ Stroop interference } \\
\hline $\begin{array}{l}\text { score } \\
\text { TOL total score }\end{array}$ & $\begin{array}{l}-0.05 \\
15.13\end{array}$ & $\begin{array}{l}4.94 \\
3.04\end{array}$ & $\begin{array}{l}-2.63 \\
19.31\end{array}$ & $\begin{array}{l}4.78 \\
3.86\end{array}$ & $\begin{array}{l}-0.33 \\
21.69\end{array}$ & $\begin{array}{l}4.70 \\
3.00\end{array}$ \\
\hline
\end{tabular}

\section{Post-Experimental Interview}

All children were able to recall the instructions of the event-based prospective memory task with only one prompt (i.e., "Other than drawing pictures and naming the cards, was there anything else you were asked to do?"), indicating that prospective forgetting was not due to retrospective failures.

Retrospective Memory Task

Table 2 shows mean number of animals remembered from the last pack of cards by group. The 6-8 group showed lower scores than either the 9-10 group or 1112 group, but the 9-10 and 11-12 groups appeared similar. A onemway ANOVA showed there was a significant effect for Group, $F(2,44)=6.99, p<.01$. Planned comparisons showed that the 6- to 8-yearmolds recalled significantly fewer animals than the 9 - to 10 -year-olds, $t(44)=3.41, p<.001$, and the 11 - to 12 -yearolds, $t(44)=3.12, p<.01$. However, there were no differences in recall between the 9-10-year-olds and 11- to 12-year-olds, $t(44)=-0.29, p>05$. 
Executive Function Tasks

Stroop. Table 2 shows group comparisons on the mean Stroop interference scores. The 9-10 group appeared to perform slightly worse on focused attention and inhibition control than the other groups. However, a one-way ANOVA showed that there were no significant differences from group to group, $F(2,44)=1.36, p$ $>.05$, and planned comparisons confirmed this.

TOL. Table 2 shows group comparisons on mean total scores on the TOL. The 6-8 group showed lower total scores than the 9-10 and 11-12 groups, and the 910 group showed lower scores than the 11-12 group. A one-way ANOVA showed significant overall group differences, $F(2,44)=15.28, p<.01$. Planned comparisons showed that the 6-8-year olds were poorer at planning than 9 w to 10 -year-olds, $t(44)=3.51, p<.001$, and 11 - to 12-year-olds, $t(44)=5.51, p<.001$. Furthermore, the 9 - to 10 -year-olds were poorer at planning than the 11 - to 12 year-olds, $t(30)=2.00, p<.05$.

\section{Executive Function/Prospective-Memory Relationships}

Results of zero-order correlations between the tests of memory and the Stroop and TOL are shown in Table 3. No significant correlations were found between the Stroop interference score and scores on either of the prospeciive memory tasks. The score on the TOL, however, was correlated significantly with both prospective memory tasks and the retrospective memory task. The event-based prospective memory task was significantly correlated with the retrospective memory task. However, Table 4 shows that when age was partialled out, no significant correlations existed between the variables of interest. ${ }^{1}$

\section{Estimated $1 Q$}

A one-way ANOVA showed no significant differences in estimated IQ across the groups, $F(2,44)=2.04, p>.05$. However, planned comparisons showed a significant difference between the 6-8 and 9 - 10 groups, $t(44)=-2.00, p<.05$. No significant differences were found between the 6-8 and 11-12 groups, $t(44)=$ $1.34, p>.05$, or the $9-10$ and 11-12 groups, $t(44)=0.67, p>05$. Because of the significant estimated IQ differences between the youngest and middle groups, the main analyses for all memory measures and executive functions were repeated using estimated $I Q$ as a covariate. Results are not reported because the covariate was not significant, and the original results remained largely unchanged.

\section{Discussion}

In general, age effects were found on the time-based task, which is consistent with those found by Kerns (2000). However, the results were not entirely consistent with the specific hypothesis, in that although the $6 m 8$ group ran out of fuel more than the 9-10 and 11-12 groups, there were no significant differences between the 9-10 and 11-12 groups. Consistent with Kerns's findings, 6- to 8year olds monitored the fuel as well as the older groups. Age effects were found on the event-based prospective memory task, but, again, the results were not 
Table 3. Zero-Order Correlation Coefficients Between Various Measures of Memory and Executive Function

\begin{tabular}{|c|c|c|c|c|c|c|c|}
\hline & $\begin{array}{l}\text { Fuel } \\
\text { Runout }\end{array}$ & $\begin{array}{l}\text { Fuel } \\
\text { Check }\end{array}$ & $\begin{array}{l}\text { Cards } \\
\text { Hidden }\end{array}$ & Recall & Stroop & TOL & $\begin{array}{l}\text { Estimated } \\
\text { IQ }\end{array}$ \\
\hline \multicolumn{8}{|l|}{ Fuel } \\
\hline Runout & - & $-.48^{* *}$ & -.20 & -.16 & .05 &. $.40^{\star \star}$ & -.17 \\
\hline \multicolumn{8}{|l|}{ Fuel } \\
\hline Check & & - & -.04 & .01 & .03 & .10 & .14 \\
\hline \multicolumn{8}{|l|}{ Cards } \\
\hline Hidden & & & - & $.39^{* *}$ &. .16 & $.48^{* *}$ & $.30^{\star}$ \\
\hline Recall & & & & - & -.24 & .26 & .26 \\
\hline Stroop & & & & & - & -.17 &. .04 \\
\hline TOL & & & & & & - & $.38^{* *}$ \\
\hline
\end{tabular}

Table 4. Partial Correlation Coefficients with Age Controlled, Between Measures of Memory and Executive Function

\begin{tabular}{|c|c|c|c|c|c|c|c|}
\hline & $\begin{array}{l}\text { Fuel } \\
\text { Runout }\end{array}$ & $\begin{array}{l}\text { Fuel } \\
\text { Check }\end{array}$ & $\begin{array}{l}\text { Cards } \\
\text { Hidden }\end{array}$ & Recall & Stroop & TOL & $\begin{array}{l}\text { Estimated } \\
\text { IQ }\end{array}$ \\
\hline \multicolumn{8}{|l|}{ Fuel } \\
\hline Runout & - & $-.46^{* *}$ & .08 & .02 & .03 &. .15 & -.10 \\
\hline \multicolumn{8}{|l|}{ Fuel } \\
\hline Check & & - & -.15 &. .05 & .04 & -.01 & .12 \\
\hline \multicolumn{8}{|l|}{ Cards } \\
\hline Hidden & & & - & .22 &. .16 & .15 & .25 \\
\hline Recall & & & & - & -.24 & -.01 & .21 \\
\hline Stroop & & & & & - &. .19 & -.04 \\
\hline TOL & & & & & & - & $.35^{*}$ \\
\hline
\end{tabular}

entirely consistent with the specific hypothesis. While the 6-8 group remembered to hide fewer cards than the $9-10$ and $11-12$ groups, the 9-10 group was also significantly poorer at remembering than the 11-12 group. TOL performance 
improved as a function of maturity, but the Stroop did not. Finally, it was found that the TOL was significantly correlated with both prospective memory tasks, but not with the retrospective memory task. However, the Stroop did not correlate with any other measure.

Given that time-based tasks are understood to be highly resource demanding, it was surprising that the 9-10-year-olds and 11-12-year-olds remembered to refuel their cars to the same degree. It was expected that three developmental stages would have emerged: 6-8 years, 9-10 years, and 11-12 years. However, it was found that by 9 years of age, the children had already developed the capacity to monitor and refuel their cars as well as those up to 12 years of age.

Children may quickly learn to self-monitor time during lunch hours and when given school work that needs to be completed in a set period of time. Moreover, these days the use of computer games by children is pervasive and some of the games involve complex rules that require the integration of information. Perhaps by 9- to 10-years, children are quite practised at these types of skills. In fact, there is evidence that skills learning, which is a type of implicit memory (Squire, 1992), is quite well developed from 3 years of age (Greenbaum \& Graf, 1989; Parkin \& Streete, 1988). Implicit memory is known to be mediated by subcortical regions of the brain (e.g., basal ganglia, Saint-Cyr \& Taylor, 1992), and posterior regions (Gabrieli, Fleischman, Kearne, \& Reminger, 1995), which are known to develop earlier than prefrontal regions (Lehr, 1990) that are thought to be implicated in prospective remembering (West, 1996).

Furthermore, practice effects have been reported in prospective memory research in ageing participants (Maylor, 1996), where they were found to quickly learn the negative consequences of forgetting, and adapt their responses. Maylor conjectured that it is adaptive to cope with situations by creating alternative behaviours, which result in more positive outcomes. As an alternative explanation for the 9-10-year-olds remembering to refuel as often as the 11-12year-olds, it may be that by 9 to 10 years of age, children, with practice, can also learn to adapt their behaviour to avoid negative consequences.

The lack of age effects in fuel checking concurs with Kerns's (2000) results and showed that 6- to 8-yearmolds were as vigilant as the two older groups in monitoring. However, given that the youngest group's monitoring was as good as the older groups', but that their refueling was not, indicated that at 6 to 8 years children still do not have the cognitive capacity to monitor in a strategic way.

Given that event-based tasks are believed to be less cognitively demanding than time-based, and that Kvavilashvili and colleagues (2001) did not find strong age effects in 4- to 7-year-olds, it was unexpected that the age effects were so strong on the current event-based task. What was particularly surprising was that 9- to 10-year-olds were significantly less able to remember to hide the cards than the 11- to 12-year-olds. Perhaps the subtle adaptation on the current task was sufficient to increase the cognitive load and task difficulty above than in the Kvavilashvili et al. task. In altering the pictures in the ongoing task from everyday items to animals, so that animals were in both the ongoing and 
prospective components, the prospective cues may have been less distinctive. In fact, cue distinctiveness has been found to assist prospective memory (Einstein \& McDaniel, 1990; Ellis, 1996). By contrast, when the cues are relatively indistinguishable from the ongoing task stimuli, they are weaker, and therefore take more cognitive awareness to be recognised. Perhaps this level of cognitive awareness is dependent on the availability of cognitive resources that younger children, up to the age of 10 years still do not possess.

Two other issues may explain some of the differences between the current results and those of Kvavilashvili and associates (2001). One, this study used a wider age range of children, which may have made the current study more sensitive to subtle changes that occur in cognition with maturity. Second, Kvavilashvili et al. did not report comparing IQs, so it is conceivable that their youngest children were as bright or brighter than their older children, which could account for the lack of age effects.

Although direct comparisons could not be made between the event- and time-based tasks, there is evidence from the current results that event-based tasks are not necessarily less resource demanding than time-based. Extra weight is given to this conclusion by the results of the correlations that showed stronger positive relationships between the TOL and the event-based task than between the TOL and the time-based task. Given that planning, as measured by the TOL, is believed to be mediated by the brain's prefrontal cortex, and that prospective memory comprises several processes similar to planning, that is, forming intentions (Shimamura, Janowsky, \& Squire, 1991), by implication, some eventbased tasks may call on higher order processes mediated by the prefrontal cortex as much as, or in this case, more than, time-based tasks.

The lack of age effects on the Stroop interference performance was inconsistent with Kerns's (2000) findings. It is difficult to explain these results. Possibly, the small sample size gave insufficient power for an age effect to emerge. Similarly, unexpectedly, the Stroop score was not significantly correlated with scores on either of the prospective memory tasks, and, again, may be the result of methodological or sampling issues in the current study.

The pattern of performance on the recall test matched that of the timebased prospective task where the youngest group was poorer than the middle and oldest groups, but the middle group was on a par with the older. However, the pattern did not match the event-based performance. These results are partly consistent with the findings by Kvavilashvili et al. (2001), who found that there were strong age effects on the retrospective memory task between 45- and 7-year-olds, but not on their event-based prospective memory task. They concluded that this indicated that prospective memory developed soooner in children than retrospective memory. However, the current findings do not support that conclusion. Here, the children showed strong age effects on the eventwbased task, such that each age group was consistently poorer than the age group above, which was the oppostite of what was found by Kvavilashvili et al. The alteration in the ongoing task in the current study and the use of a wider age range may have made a big difference to the two studies' 
results. Whatever the explanation for the differences, these results suggest on the one hand, that prospective memory does not necessarily develop sooner than retrospective. In fact, there is evidence that the area of the brain involved in prospective remembering (i.e., the prefrontal cortex) develops later than other parts of the brain (Lehr, 1990), including the temporal lobe, which is believed to be the anatomical substrate of retrospective memory (Nissen, 1992; Sain-Cyr \& Taylor, 1992). Moreover, the event-based prospective task and retrospective task differences give additional support for the complexity of event-based prospective memory. In particular, it seems not to matter so much whether a prospective task is time- or event-based, but how cognitively demanding that task is in terms of what resources children have at their disposal.

The current study overcame limitations of previous research by incorporating both time- and event-based prospective memory tasks, retrospective memory tasks, and tests of executive function. It also separated children from a wide age range into subgroups to better detect subtle developmental trends. The key findings indicated that different aspects of prospective and retrospective memory have different developmental trajectories, and that the degree of cognitive demand imposed by a task makes a difference to how well children of different ages can remember.

There were a number of limitations in this study. First, the sample size was small, possibly masking effects that otherwise would have been significant. Second, the retrospective memory measure may have underestimated the recall capacities of the children. This may have occurred because the retrospective measure was an incidental task, where the children were not told to remember the pictures before completing the picture naming. Similarly, the recall task may have been bound up too closely with the highly resource demanding eventbased prospective task. In having to focus so much on the ongoing task, interrupt the process, and remember to hide the cards, the children may have had insufficient cognitive resources left to encode the items for short-term recall.

Future research should use a larger sample of children, and incorporate a more distinctive retrospective-memory task. Follow ups should also examine more closely the effects of task complexity on childhood prospective memory. Children of different ages might be compared on tasks in which the cognitive loads of the ongoing tasks are manipulated more precisely and systematically. For example, ongoing tasks might comprise three levels: low, medium, and high cognitive demand. Furthermore, the relationships between prospective memory and executive functions should be explored further because they help identify the mental processes common (or not) to both, and may lead to the identification of the neural substrates of these processes. Further knowledge, in turn, may help develop rehabilitation strategies for those with prospective memory and executive function impairments. In terms of normal, everyday learning, the findings suggest that teachers and parents need to be careful when asking younger children to remember future tasks to keep them simple, 
use distinctive cues, give instructions at times when the children's minds are relatively free to encode the information effectively, and to remind them. In addition, these results make it clear how important planning is in fulfilling future intentions, and suggest that planning might be taught explicitly from a young age.

\section{Address for correspondence}

Associate Professor David Shum

School of Psychology

Griffith University

Nathan, Queensland Australia 4111

Phone: 61738753370

E-mail: d.shum@griffith.edu.au

\section{References}

Anderson, V., Northam, E., Hendy, J., \& Wrennall, J. (2001). Developmental Neuropsychology: A clinical approach. East Sussex, UK: Psychology Press Ltd.

Ceci, S. J., \& Bronfenbrenner, U. (1985). "Don't forget to take the cupcakes out of the oven": Prospective memory, strategic time monitoring, and context. Child Development, 56, 152-164.

Einstein, G. O., \& McDaniel, M. A. (1990). Normal aging and prospective memory. Journal of Experimental Psychology: Learning, Memory and Cognition, $16,717-726$.

Ellis, ]. (1996). Prospective memory or the realization of delayed intentions: A conceptual framework for research. In M. Brandimonte, G. Einstein \& M. McDaniel (Eds.), Prospective memory: Theories and Applications (pp. 1-22). Mahwah, NJ: Lawrence Erlbaum Associates.

Ellis, J. \& Kvavilashvili, L. (2000). Prospective memory in 2000: Past, present, and future directions. Applied Cognitive Psychology, 14, S1-59.

Gabrieli, J. D. E., Fleischman, D. A., Kearne, M. M., \& Reminger, S. L. (1995). Double dissociation between memory stystems underlying explicit and implicit memory in the human brain. Psychological Science, 6, 76 82 .

Golden, C. J., Espe-Pfeifer, P., \& Wachsler-Felder, J. (2000). Neturopsychological interpretations of objective psychological tests. NY: Kluwer Academic/Plenum Publishers.

Hanes, K. R., Andrewes, D. G., Smith, D. J., \& Pantelis, C. (1996). A brief assessment of executive control dysfunction: Discriminant validity and homogeneity of planning, set shift, and fluency measures. Archives of Clinical Neuropsychology, 18, 185-191.

Kerns, K. A. (2000). The CyberCruiser: An investigation of development of prospective memory in children. Journal of International Neuropsychological Society, 6, 62-70.

Kvavilashvili, L., Messer, D. J., \& Ebdon, P. (2001). Prospective memory in children: The effects of age and task interruption. Developmental Psychology, $37,418-430$. 
Lehr, E. (1990). Psychological management of traumatic brain injuries in children and adolescents (Rehabilitation Institute of Chicago Procedure Manual). Rockville, MD: Aspen Publishers, Inc.

Levin, H. S., Song, J., Scheibel, R. S., Fletcher, J. M., Harward, H., Lilly, M., \& Goldstein, F. (1997). Concept formation and problem solving following closed head injury in children. Journal of the International Netropsychological Society, 3 , 598-607.

Lezak, M. D. (1982). The problem of assessing executive functions. International Journal of Psychology, 17, 281.

Maujean, A., Shum, D., \& McQueen, R. (2003). The effect of cognitive demand on prospective memory in individuals with traumatic brain injury. Brain Impainment, 4 ,

Maylor, E. A. (1996). Does prospective memory decline with age? In M. Brandimonte, G. O. Einstein, \& M. A. McDaniel (Eds.), Prospective memory: Theory and applications (pp. 115-141). New Jersey: Lawrence Erlbaum Associates Lutd.

Nissen, M. J. (1992). Procedural and declarative learning: Distinctions and interactions, In L. R. Squire \& N. Butters (Eds.), Neuropsychology of memory (2nd ed., pp. 203-210). New York: The Guilford Press.

Saint-Cyr, J. A., \& Taylor, A. E. (1992). The mobilization of procedural learning: The "key signature" of the basal ganglia. In L. R. Squire \& N. Butters (Eds.), Neuropsychology of memory (2nd ed., pp. 188-202). New York: The Guilford Press.

Shallice, T. (1982). Specific impairments of planning. Philosophical Transactions of The Royal Society of London B, 298, 199-209.

Shimamura, A. P., Janowsky, J. S. \& Squire, L. R. (1991). What is the role of frontal lobe damage in memory disorders? In H. S. Levin, H. M. Eisenburg, and A. L. Benton (Eds.), Frontal lobe function and dysfunction (pp. 173-195). NewYork: Oxford University Press.

Shum, D. H. K., McFarland, K. A., \& Bain, J. D. (1990). Construct validity of eight: tests of attention: Comparison of normal and closed head injured samples. The Clinical Neuropsychologist, 4, 141-162.

Shum, D., Short, L., Tunstall, I., O'Gorman, J. G., Wallace, G., Shephard, K., \& Murray, R. (2000). Performance of children with traumatic brain injury on a 4disk version of the Tower of London and the Porteus Maze. Brain and Cognition, 44, 59-62.

Squire, L. R. (1992). Declarative and nondeclarative memory: Multiple brain systems supporting learning and memory. Journal of Cognitive Neuroscience, 4 , 232-243.

The Psychological Corporation (1999). Wechsler Abbreviated Scale of Intelligence. San Antonia, CA: Author.

Tunstall, J. (19999). Improving the utility of the Tower of London: A Neuropsychological test of planning. Unpublished M Phil: Griffith University, Brisbane, Australia.

Uttl, B., \& Graf, P. (1997). Color word stroop test performance across the adult life Span. Journal of Clinical and Experimental Neuropsychology, 19, 405-420. 
West, R. L. (1988). Prospective memory and aging. In M. M. Gruneberg, P. E. Morris \& R. N. Sykes (Eds.), Practical aspects of memory: Current Research and issues, Vol 1 (pp. 348-353). Chichester, UK: John Wiley \& Sons.

\section{Note}

1 predicted scores on the three measures of prospective memory as revealed by a series of hierarchical multiple regressions with age entered at step 1 , executive function scores entered at step 2, and the retrospective memory measure entered at step 3 , showed similar results to the correlation analyses. 